\title{
Controlling circular polarization of light emitted by quantum dots using chiral photonic crystal slabs
}

\author{
S. V. Lobanov, ${ }^{1,2,3}$ S. G. Tikhodeev,${ }^{1,4,5,{ }^{*}}$ N. A. Gippius,,${ }^{3,1}$ A. A. Maksimov, ${ }^{4}$ E. V. Filatov, ${ }^{4}$ I. I. Tartakovskii, ${ }^{4}$ \\ V. D. Kulakovskii, ${ }^{4}$ T. Weiss, ${ }^{6}$ C. Schneider, ${ }^{7}$ J. Geßler, ${ }^{7}$ M. Kamp, ${ }^{7}$ and S. Höfling ${ }^{7,8}$ \\ ${ }^{1}$ A. M. Prokhorov General Physics Institute, Russian Academy of Sciences, Vavilova Street 38, Moscow 119991, Russia \\ ${ }^{2}$ School of Physics and Astronomy, Cardiff University, Cardiff CF24 3AA, United Kingdom \\ ${ }^{3}$ Skolkovo Institute of Science and Technology, Novaya Street 100, Skolkovo 143025, Russia \\ ${ }^{4}$ Institute of Solid State Physics, Russian Academy of Science, Chernogolovka 142432, Russia \\ ${ }^{5}$ M. V. Lomonosov Moscow State University, Leninskie Gory 1, Moscow 119991, Russia \\ ${ }^{6} 4$ th Physics Institute and Research Center SCoPE, University of Stuttgart, Stuttgart D-70550, Germany \\ ${ }^{7}$ Technische Physik, Physikalisches Institut and Wilhelm Conrad Röntgen Research Center for Complex Material Systems, \\ Universität Würzburg, D-97074 Würzburg, Germany \\ ${ }^{8}$ SUPA, School of Physics and Astronomy, University of St Andrews, St Andrews, KY16 9SS, United Kingdom
}

(Received 25 September 2015; published 23 November 2015)

\begin{abstract}
We study the polarization properties of light emitted by quantum dots that are embedded in chiral photonic crystal structures made of achiral planar GaAs waveguides. A modification of the electromagnetic mode structure due to the chiral grating fabricated by partial etching of the waveguide layer has been shown to result in a high circular polarization degree $\rho_{c}$ of the quantum dot emission in the absence of external magnetic field. The physical nature of the phenomenon can be understood in terms of the reciprocity principle taking into account the structural symmetry. At the resonance wavelength, the magnitude of $\left|\rho_{c}\right|$ is predicted to exceed $98 \%$. The experimentally achieved value of $\left|\rho_{c}\right|=81 \%$ is smaller, which is due to the contribution of unpolarized light scattered by grating defects, thus breaking its periodicity. The achieved polarization degree estimated removing the unpolarized nonresonant background from the emission spectra can be estimated to be as high as $96 \%$, close to the theoretical prediction.
\end{abstract}

DOI: 10.1103/PhysRevB.92.205309

PACS number(s): 71.36.+c, 42.65.Pc, 42.55.Sa

\section{INTRODUCTION}

The possibility to control the polarization state of radiation from quantum emitters has drawn attention of researchers in recent years as it opens various important applications in spin optoelectronics, quantum information technology, chiral synthesis, and sensing. A common way for polarization conversion and rotation is the use of wave plates of birefringent materials and optical gratings. An imbalance between the left- and right-circularly polarized photons takes place in chiral materials with nonequivalent left- and right-circularly polarized electromagnetic field modes [1-4]. The imbalance in semiconductors is usually reached by applying a static magnetic field that disturbs the time-reversal symmetry and leads to a splitting of the left- and right-circularly polarized modes.

Advances in nanoscale fabrication have made it possible to realize artificial photonic structures with desired symmetry and density of the environmentally allowed electromagnetic modes, which allows us to control the spontaneous emission rate of emitted light, its radiation pattern, and direction. Moreover, the fabrication of chiral nanostructures from achiral semiconductor materials opens the possibility to control the polarization of emitted light [5,6], as the polarization effects are determined by the overall structure symmetry [7]. This method has considerable advantages in comparison with the others as it is compatible with semiconductor technology and allows us to fabricate nanoscale devices (thickness of a

*Corresponding author: tikh@gpi.ru conventional quarter-wave plate is much larger) with a simple operation (external magnetic field application is not easy).

Recently Konishi et al. [5] fabricated a simple chiral nanostructure that consisted of InAs quantum dots (QDs) embedded in the waveguide region of a planar GaAs based dielectric waveguide coupled to a dielectric chiral photonic crystal slab (CPCS), and obtained a degree of circular polarization of QD emission $\rho_{c}=26 \%$. A significantly higher value of $\rho_{c}$ of QD emission (up to $70 \%$ in the direction normal to the structure plane and up to $81 \%$ at some angle) was experimentally achieved from a planar semiconductor microcavity with chiral partially etched top mirror [8], but this structure is far more complex. The degree of circular polarization of emission from a chiral structure made of achiral semiconductors depends strongly on its geometry and the radiation frequency, which allows one to optimize the structures. Recent numerical simulations [8,9] show that values of $\rho_{c}$ as high as 98 and $99 \%$ in waveguide and microcavity structures, respectively, can be reached by their optimization.

In this paper, we have experimentally investigated the polarization of emission of InAs QDs embedded in a planar GaAs based dielectric waveguide coupled to a dielectric chiral photonic crystal slab with subwavelength period in order to reach the predicted high values of $\rho_{c}$. The structure geometry has been optimized on the base of the computation of the QDs radiation performed using an oscillating point dipole model $[9,10]$. A circular polarization degree of InAs QD emission in the finite (NA less than 0.02) aperture as high as $81 \%$ is experimentally realized in fabricated optimized structures. The achieved value is found to be limited by the unpolarized 
scattered light background caused by the fluctuations in the periodic potential due to the size and shape imperfections of etched rectangular pillars in the chiral layer. It is worthwhile to note that the magnitude of $\rho_{c}$ obtained from the spectra with canceled background due to the lattice imperfection reaches $96 \%$. Thus, the considered very simple chiral structure made of conventional achiral semiconductor materials using the chiral morphology effect can enable the fabrication of compact solid-state circularly polarized light-emitting devices with future progress in fabrication technology.

In addition, we clarify the physical nature of the phenomenon. Based on the reciprocity principle and the symmetry of the structure, we show the possibility to enhance and filter out one circularly polarized light component from the originally unpolarized QD emission. This is a consequence of the Fabry-Perot resonances between propagating photon modes in the chiral photonic crystal slab. We show that the circular polarization efficiency of such a chiral filter has to oscillate with its thickness, and the CPCS in the optimized structure is actually a half-wave plate. Its thickness can be made however much less than in the case of using natural optically active materials with a weak chirality, of the order of the emitted light wavelength $\lambda$ only. It happens because in a dielectric CPCS with a high contrast of dielectric constants it becomes possible to achieve a large difference between wave numbers of different propagating modes, of the order of $k_{0}=2 \pi / \lambda$.

We would like to add that recently, a possibility to control deterministically the direction of photon emission (depending on the handedness of its circular polarization) by a QD inside a chiral photonic crystal waveguide has been demonstrated [11]. Our structure with randomly distributed QDs may open an alternative way to this goal, being not sensitive to the exact positioning of the emitting QDs inside the structure.

The paper is organized as follows: The experimental details and the sample are described in Sec. II. The experimental results are described in Sec. III. The physical nature of the effect is discussed in Sec. IV. Section V contains the concluding remarks.

\section{EXPERIMENT}

The epitaxial layer structure, which formed the basis of this study, consisted of a 691-nm-thick GaAs membrane with a single layer of embedded randomly distributed InAs quantum dots [Fig. 1(a)]. The membrane is grown on top of a 1- $\mu$ m-thick AlGaAs buffer layer. We fabricated an array of nanopillars into the upper nominally 428-nm-thick part of the membrane layer via electron-beam lithography and reactive ion etching. The nanopillars were arranged into a square lattice of unit cells consisting of four rectangular pillars, each rotated by $90^{\circ}$ with respect to its nearest neighbors. The lattice period $p$ was varied in the range $0.7-1.1 \mu \mathrm{m}$. The unit cell is shown in Fig. 1(b). The distance between pillars was $p / 5$, their sides were $p / 5$ and $2 p / 5$, respectively. Both right and left (mirrored) twisted pillars were manufactured. (For definiteness, we call hereafter the structures shown in Figs. 1 and 2 right twisted.) The thickness of the GaAs waveguide layer $\left(\epsilon_{\mathrm{GaAs}}=12.42\right)$ containing a plane with QDs was $263 \mathrm{~nm}$. The plane with QDs was located $100 \mathrm{~nm}$ above its bottom. The lower $\mathrm{Al}_{0.7} \mathrm{Ga}_{0.3} \mathrm{As}$
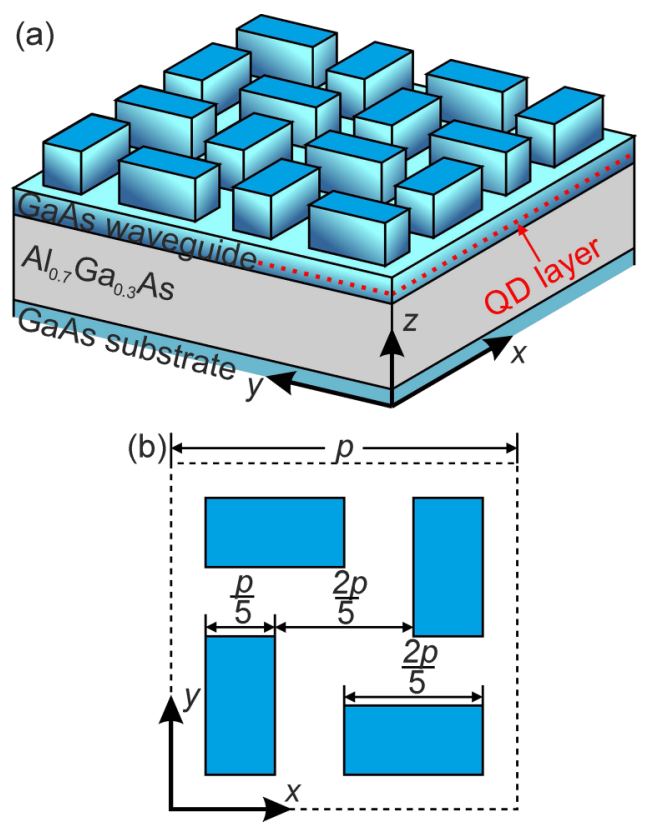

FIG. 1. (Color online) (a) Schematics of a structure with GaAs/air chiral photonic crystal slab, GaAs waveguide layer with an embedded layer of QDs, and a $\mathrm{Al}_{0.7} \mathrm{Ga}_{0.3}$ As cladding layer on $\mathrm{GaAs}$ substrate; (b) top view of one period of the right (or counterclockwise) twisted structure.

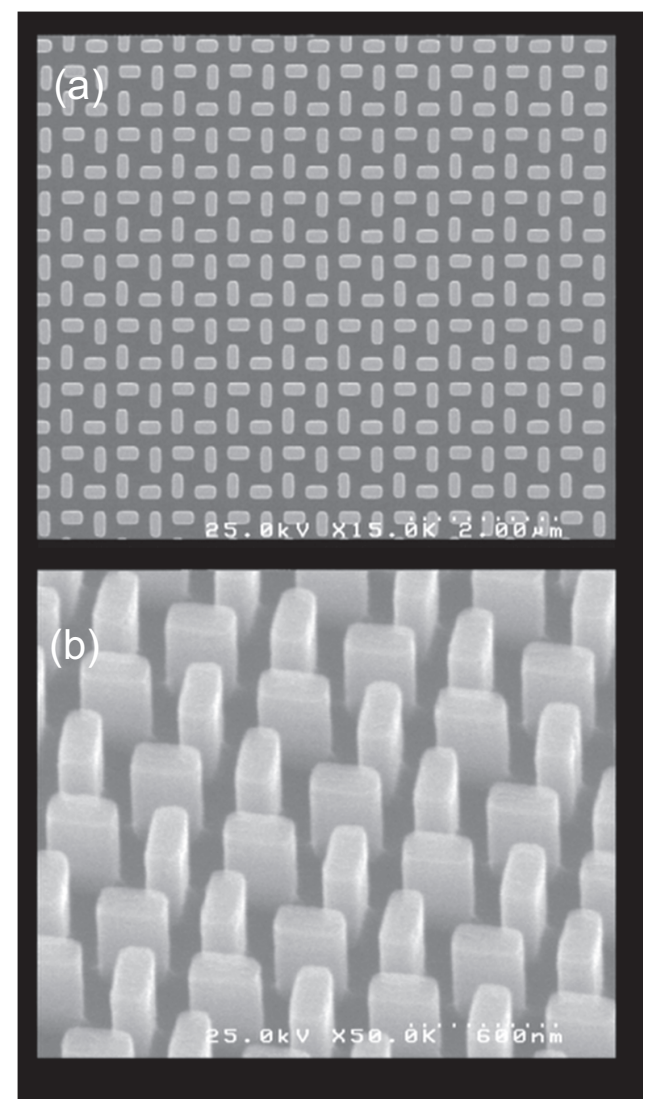

FIG. 2. Representative scanning electron microscopy (SEM) pictures of the right-twisted chiral photonic crystal fabricated from the GaAs-based waveguide, top (a) and side (b) view. 

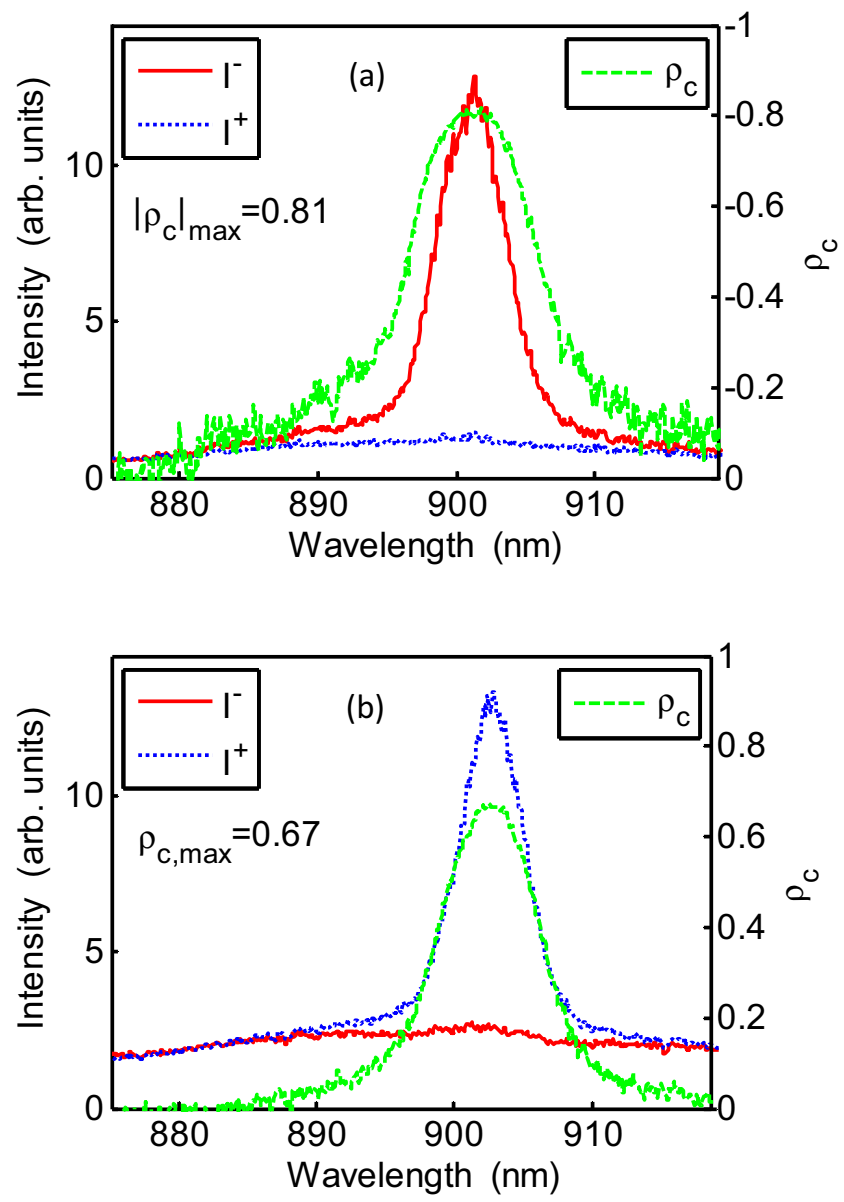

FIG. 3. (Color online) Experimental circularly polarized spectra of QD emission from the fabricated chiral waveguide structures with (a) the right- and (b) the left-twisted rectangular pillars. Green dashed lines show the corresponding circular polarization degree $\rho_{c}$.

$\left(\epsilon_{\mathrm{AlGaAs}}=9.66\right)$ buffer layer was $1 \mu \mathrm{m}$ thick. The whole system was grown by molecular-beam epitaxy on a GaAs substrate. Representative SEM pictures of the right-twisted chiral photonic layer are shown in Fig. 2.

The sample was at a temperature of $5 \mathrm{~K}$ in the insert of an optical cryostat. The luminescence polarization was analyzed by a quarter wave retarder and linear polarizers. The angle resolution of $0.5^{\circ}$ is ensured by a small aperture. The circular polarization degree of the luminescence from the structure prior to etching was less than $0.1 \%$.

\section{EXPERIMENTAL RESULTS}

Experimental circularly polarized spectra of QD emission are shown in Fig. 3 (left axis) for left and right-twisted rectangular pillars. The green lines in Fig. 3, right axes, show the resulting spectra of the circular polarization degree of emission,

$$
\rho_{c}=\frac{I^{+}-I^{-}}{I^{+}+I^{-}},
$$

where $I^{ \pm}$is the emission intensity in right/left circular $\sigma^{ \pm}$ polarization [12]. The spectra are recorded in the direction

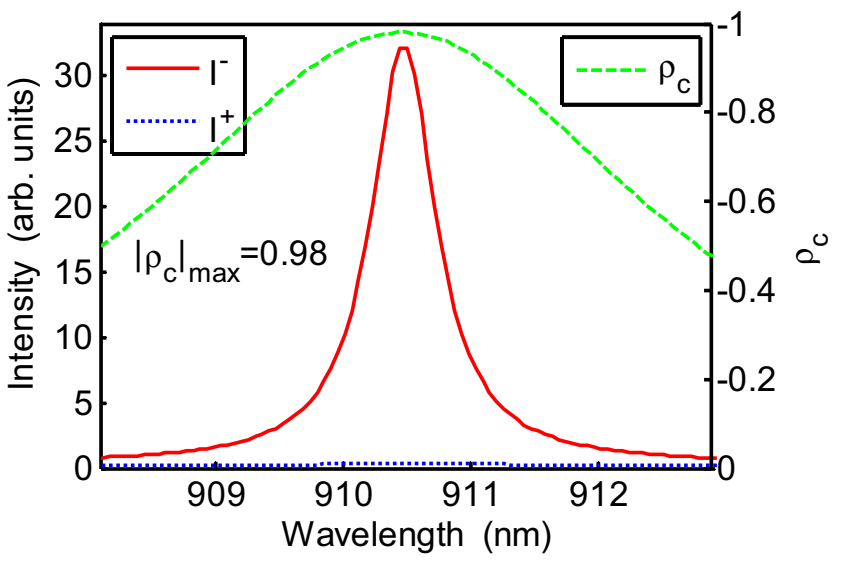

FIG. 4. (Color online) Calculated circularly polarized spectra and circular polarization degree of QD emission from the chiral waveguide structure with the right-twisted pillars. The sign of circular polarization is opposite for the left-twisted pillars. The emission intensity is normalized to the emission intensity of the same oscillating dipole in vacuum (without the structure).

$z$ (perpendicular to the structure plane) with numerical aperture NA $=0.005$. For comparison, the calculated emission spectra in $\sigma^{ \pm}$polarization and $\rho_{c}$ are shown in Fig. 4 for the right-twisted structure with the same parameters. The spectra were calculated using the reciprocity principle and the optical scattering matrix method [8,9]. Compared to Ref. [9], we changed the parameters of the materials, because the current experiment is carried out at low rather than at room temperature.

Figure 3 shows that, in accordance with the calculations, the polarization of the emission line at the resonance frequency is opposite in the structures with the left- and right-twisted pillars.

The experimental and calculated dependencies in Figs. 3 and 4 are in a good qualitative agreement. A small 9-nm redshift of the calculated resonance wavelength is most probably due to slightly incorrect dielectric susceptibilities used in the calculations; the agreement can be easily improved by their fitting.

Note, however, that (i) the experimental line FWHM is about five times greater than the calculated one $(\approx 4$ and $0.8 \mathrm{~nm}$, respectively), and (ii) the line sits on a background whose magnitude is about $9 \%$ from its peak intensity whereas the background in the calculated spectra does not exceed $1 \%$. Both the line broadening and background magnitude are found to depend strongly on the pillar quality, which indicates small deviations from the ideal structure. Figure 3 shows that magnitudes of $\rho_{c}=-0.81$ and +0.67 in the waveguides with the right- and left-twisted pillars are limited by the unpolarized background. It is worthwhile to note that the magnitude of $\left|\rho_{c}\right|$ at the resonance reaches 0.96 when this unpolarized background is removed as shown in Fig. 5. This value is very close to the calculated one, which indicates that the predicted values of $\left|\rho_{c}\right| \approx 0.98$ could be reached due to further advances in fabrication technology. 


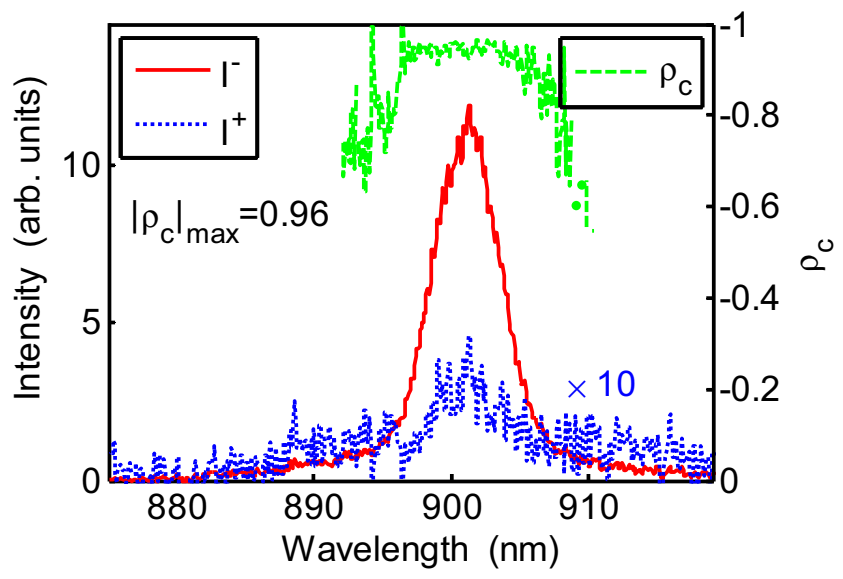

FIG. 5. (Color online) Experimental circularly polarized spectrum of QD emission from the fabricated chiral waveguide structures with the right-twisted pillars, Fig. 3(a), with subtracted unpolarized background.

\section{DISCUSSION}

\section{A. Angular dependencies of emission intensities $\rho_{c}$ and the quasiguided modes}

As already discussed [5,9], the peaks in the QD emission spectrum are due to the excitation of lossy quasiguided modes in the GaAs waveguide inside a periodically modulated structure. Their expected angular dispersion is shown in Fig. 6 , where false colors show the calculated intensity of the emission (in $\sigma^{-}$polarization) from the right-twisted structure with period $p=770 \mathrm{~nm}$ as a function of energy (wavelength) and polar angle $\theta$ in $M-\Gamma$ (diagonal) and $\Gamma-X$ directions. These Bragg resonances can be understood [13] as corresponding approximately to the folding of the dispersion of the guided

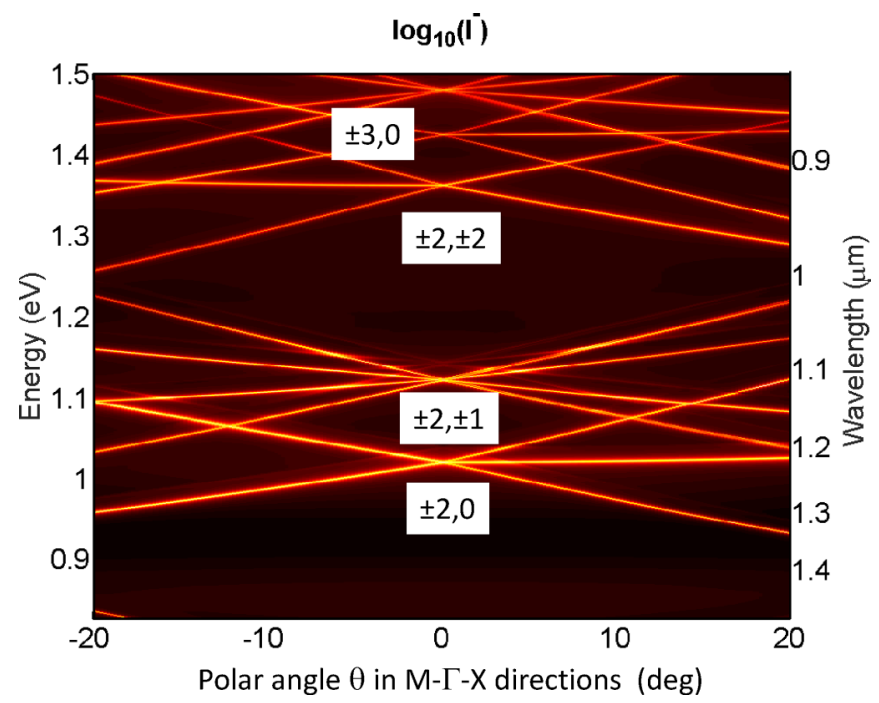

FIG. 6. (Color online) Dispersion of quasiguided modes in spatially structured GaAs waveguide. False color scale shows the calculated QD emission intensities (in left polarization, logarithmic scale) at various $k=k_{0} \sin \theta, k_{0}=\omega / c$, along $\Gamma-X$ and $\Gamma-M$ (diagonal) directions in the first BZ. Labels $l, m$ (see in the text) mark the order of the corresponding Bragg resonance in the $\Gamma$ point. modes into the 1st Brillouin zone (BZ) of the two-dimensional (2D) grating (square lattice in our case),

$$
E_{l, m}(\mathbf{k})=\frac{\hbar c}{n_{\mathrm{eff}}} \sqrt{\left(k_{x}+l K_{\mathrm{BZ}}\right)^{2}+\left(k_{y}+m K_{\mathrm{BZ}}\right)^{2}},
$$

where $\mathbf{k}=\left(k_{x}, k_{y}\right)$ is the in-plane wave vector, $K_{\mathrm{BZ}}=2 \pi / p$ is the Bragg wave number, $p$ is the period, $n_{\text {eff }}$ is the effective refractive index of the guided mode, $l$ and $m$ are integers. It appears that the resonance of interest at $\lambda_{0} \sim 901 \mathrm{~nm}$ in the current structure corresponds to a fourfold degenerate $l, m= \pm 2$ state in the $\Gamma$ point (in the scalar approximation and neglecting the energy splitting due to the point symmetry group); see Fig. 6. It is also in agreement with the analysis of Ref. [9]; see, e.g., the calculated in-plane distribution of the emission intensity in the dominant polarization in Fig. 6(b) of Ref. [9].

The degeneracy of the state $E_{ \pm 2, \pm 2}$ is lifted at $k \neq 0$. According to Eq. (2), near the $\Gamma$ point

$$
E_{l, m}(\mathbf{k})=E_{0}+\frac{\hbar c\left(l k_{x}+m k_{y}\right)}{n_{\mathrm{eff}} \sqrt{l^{2}+m^{2}}}+\mathcal{O}\left(k_{x}^{2}+k_{y}^{2}\right),
$$

where $E_{0}$ is the resonance energy in the $\Gamma$ point, $E_{0} \equiv$ $E_{ \pm 2, \pm 2}(k=0)$. Thus, in the $0-X, 0-Y$ directions, according to Eq. (3), the $( \pm 2, \pm 2)$ modes split into two degenerate doublets with nearly linear $k-$ dispersion $E-E_{0}=$ $\pm \hbar c k_{x, y} /\left(\sqrt{2} n_{\text {eff }}\right)$. In the diagonal directions, the modes with $l=m= \pm 2$ split into two singlets with nearly linear dispersion $E-E_{0}= \pm \hbar c k / n_{\text {eff }}$, whereas those with $l=-m= \pm 2$ is a degenerate parabolic doublet with an effective mass $m_{\mathrm{eff}} \sim 10^{-5} m_{e}$, where $m_{e}$ is the free-electron mass. Thus, the modes with $l=-m= \pm 2$ are nearly dispersionless at small $k \lesssim 0.2 \mu \mathrm{m}^{-1}$. As a result, the lines of equal energy at $E=E_{0}$ are two mutually perpendicular lines at $k_{y}= \pm k_{x}$ in this range of $k$. That is well seen in Figs. 7(c) and 7(d) representing the measured angular distribution of the QD emission near $\theta=0$ in two circular polarizations at the resonance wavelength.

Figures 7(a) and 7(b) and 7(e) and 7(f) show that these lines at $E \neq E_{0}$ split into doublets $k_{y}=k_{x} \pm \Delta k$ and $k_{y}=$ $-k_{x} \pm \Delta k$ in accordance with the $D_{4}$ point-group symmetry of the modes' dispersion at the $\Gamma$ point.

The measured angular distributions of the emission intensities in $\sigma^{ \pm}$polarizations and of the circular polarization degree (left panels of Figs. 7 and 8 respectively) are in a good qualitative agreement with the calculated ones displayed in the right panels. It is seen in Fig. 7 that, in agreement with the calculation, the strongest emission in the dominated $\left(\sigma^{-}\right)$polarization is at the crossing of the isoenergetic lines and reaches its maximum at $E=E_{0}$ when the state at $k=0$ becomes fourfold degenerate. According to calculations shown in Fig. 4 the QD emission intensity in the dominating left polarization at the resonance $E=E_{0}$ exceeds that in vacuum more than 30 times, whereas that in the opposite polarization is strongly (about three times) suppressed. The effect is connected to a modification of the environmentally allowed electromagnetic modes by the CPCS in $k$ space. As a consequence, it is very sensitive to the quality of the photonic crystal. Any disturbance of the potential periodicity results in the increased Rayleigh scattering contributing to $k=0$ emission in the suppressed polarization. The result is the decreased degree of circular polarization in the experiment. 

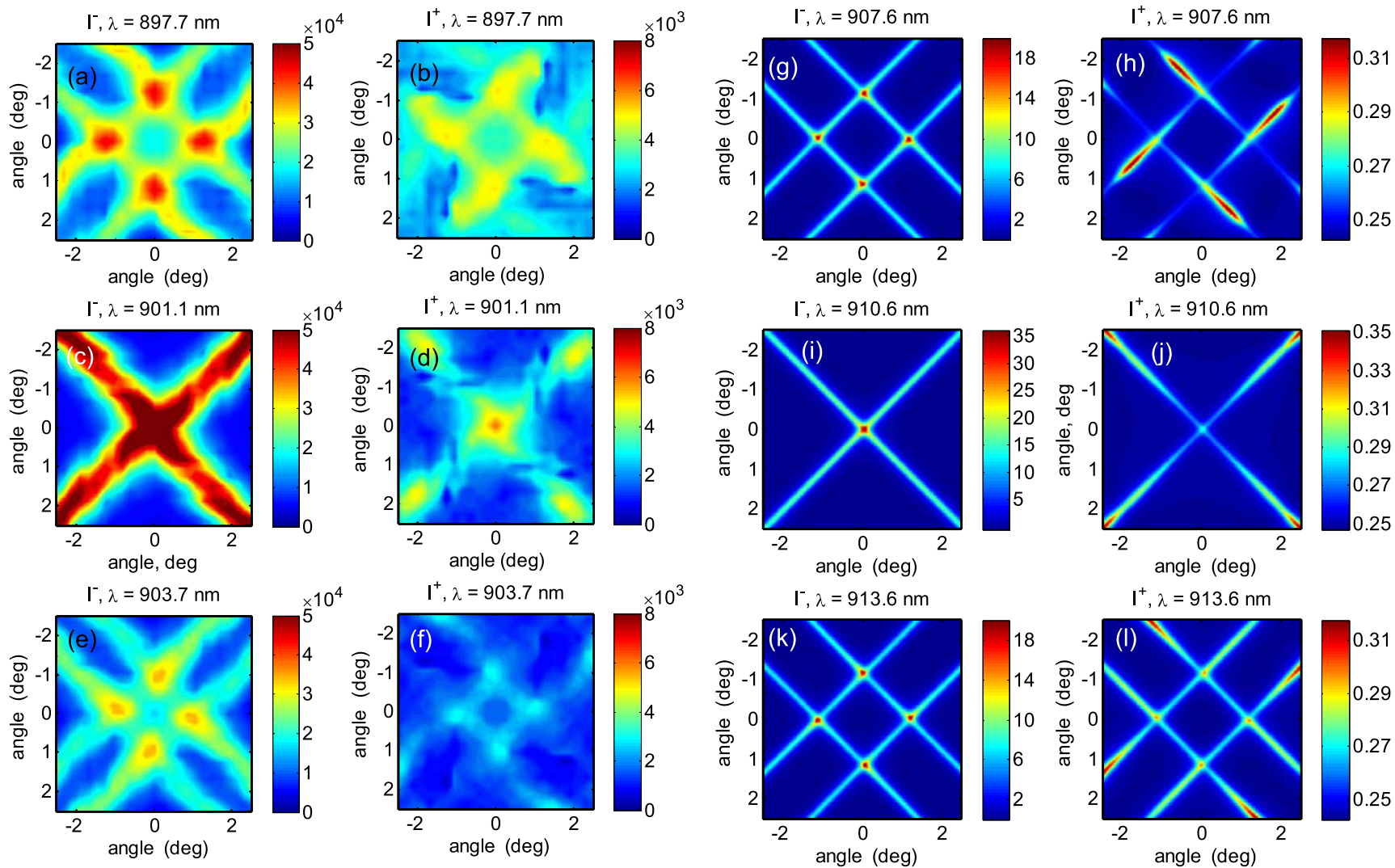

FIG. 7. (Color online) Polar plots of the measured (left panels) and calculated (right panels) angle dependencies of the QD emission intensities in left and right circular polarizations. The measured spectra were recorded for the resonance wavelength $\lambda_{0}=901.1 \mathrm{~nm}$ [panels (c) and (d)], corresponding to the $( \pm 2, \pm 2)$ Bragg resonance at $E=E_{0}$ (see text). Panels (a) and (b) show the corresponding dependencies for $\lambda_{0}-3.4 \mathrm{~nm}$, while panels (e) and (f) depict the results for $\lambda_{0}+2.6 \mathrm{~nm}$. The simulated spectra were calculated around the theoretical resonance wavelength $\lambda_{0, \text { th }}=910.5 \mathrm{~nm}$ [panels (i) and (j)]. Additionally, we show the corresponding dependencies $3 \mathrm{~nm}$ below [panels (g) and (h)] and above [panels (k) and (l)] $\lambda_{0, \text { th }}$.

\section{B. Symmetry analysis and the physical origin of circularly polarized emission}

Although the possibility to obtain nearly $100 \%$ circularly polarized emission from unpolarized emission of randomly distributed QD was already demonstrated numerically [8,9], the physical mechanism of this effect has not been yet clarified. The key property of the structure that helps us to understand this mechanism is the expected theoretically oscillatory dependence of the degree of circular polarization of emission of such structures on the thickness of the chiral photonic slab thickness, illustrated in Fig. 9. While the calculated dependencies of the emission intensities in right and left polarization $I^{ \pm}$on $L$ [see Fig. 9(a)] consist of series of at first sight incoherent peaks, the dependence $\rho_{c}(L)$ [see green line in Fig. 9(b)] looks like coherent Fabry-Perot oscillations. In what follows, based on the reciprocity and symmetry properties of our structure, we show that the chiral photonic crystal slab in the structure of interest plays the role of a wave plate. It introduces a phase shift, proportional to $\Delta K_{z} L$ ( $L$ is the CPCS thickness), between the propagating modes with a large (of the order of $k_{0}=\omega / c$ ) difference of wave numbers $\Delta K_{z}$. We will show that these modes are basically different waveguide modes in the GaAs rectangular vertical waveguides, i.e., in the building blocks of the CPCS. There is also a large constant phase shift between left and right circular polarizations, due to the absence of horizontal mirror symmetry of the photonic crystal slab with different top and bottom boundaries (air/CPCS and $\mathrm{CPCS} / \mathrm{GaAs}$, respectively). As a result, the transmission of the CPCS in left and right polarizations oscillate as a function of $L$ in the counterphase, which opens a way to reach nearly $100 \%$ polarization selectivity.

\section{Reciprocity principle for the structures with $C_{4}$ and $\mathrm{D}_{\mathbf{4}}$ symmetry}

The emission intensity of QDs can be calculated directly $[10,14-16]$ or using the electromagnetic reciprocity principle $[8,17]$. In the latter case, the emission problem of randomly distributed and randomly polarized oscillating dipoles is replaced by an illumination problem, in which the structure is illuminated by a polarized plane wave $\mathbf{E}_{\text {in }}(x, y, z)=$ $E \mathbf{e}^{j} \exp \left(i k_{0} z-i \omega t\right)$. (Hereinafter index $j$ marks the polarization state, e.g., $j=x, y$ in the case of linear polarization along $x, y$, respectively, and $j= \pm$ for right and left polarization.) Then, the resulting electric-field distribution in the layer with QD,

$$
I^{j}=\iint d x d y\left(\left|E_{x}^{j}\right|^{2}+\left|E_{y}^{j}\right|^{2}\right),
$$



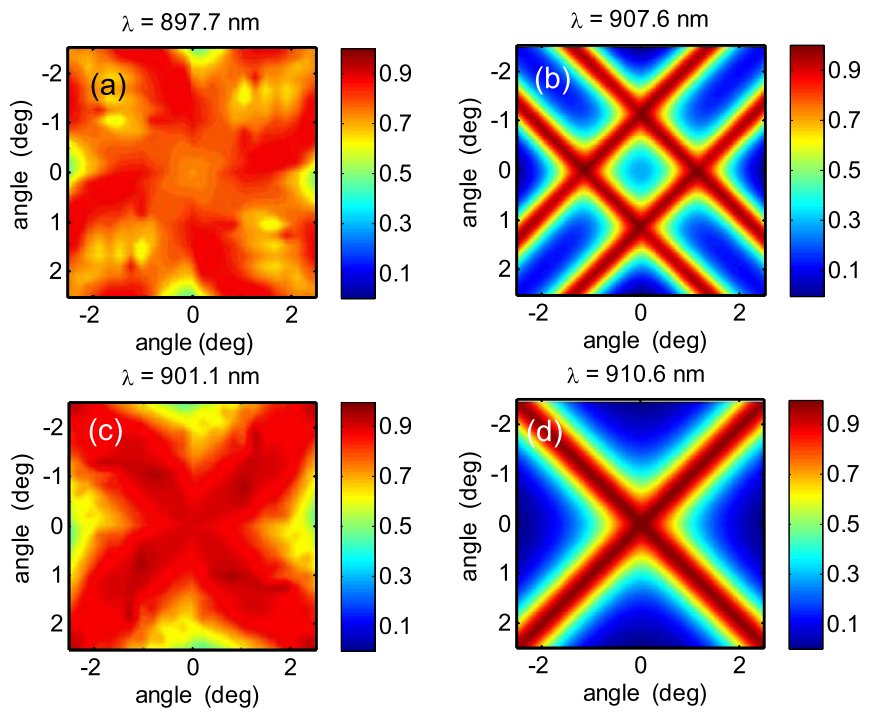

$\lambda=903.7 \mathrm{~nm}$
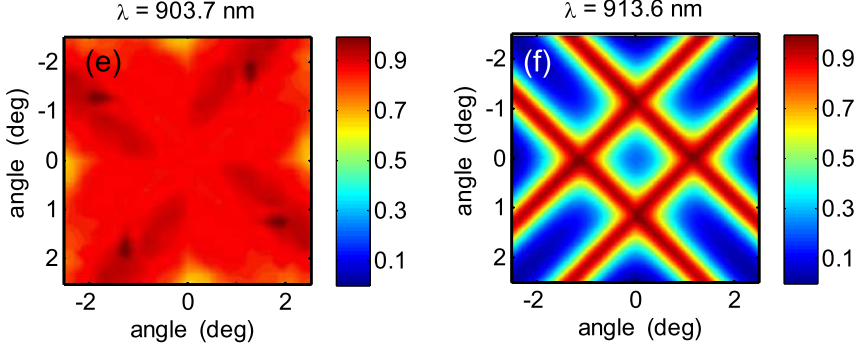

FIG. 8. (Color online) Polar plots of the measured (left panels) and calculated (right panels) angle dependencies of $\left|\rho_{c}\right|$ of the QDs emission, corresponding to the data in Fig. 7.

gives the corresponding polarized component of the QD radiation intensity [8].

The reciprocity principle helps us to understand easily why in the structure with a $C_{4}$ symmetry the emission of QD in the vertical direction cannot be linearly polarized, and why for a structure with higher symmetry (e.g., $D_{4}$ ), it cannot be also circularly polarized.

Assume $\mathbf{E}(x, y, z)$ and $\mathbf{H}(x, y, z)$ are the solutions of Maxwell's equations for the system with $C_{4}$ symmetry. Then,

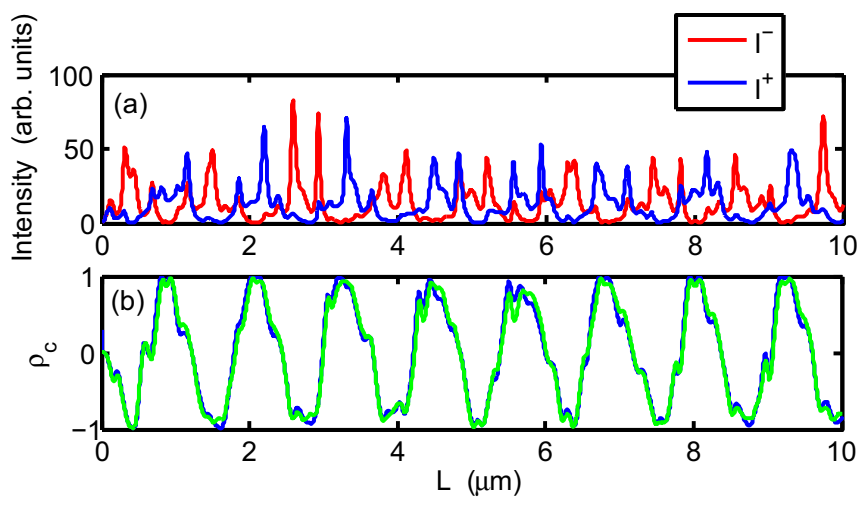

FIG. 9. (Color online) The calculated dependencies of (a) the emission intensities $I^{ \pm}$and (b) (green line) the circular polarization degree of emission $\rho_{c}$ at the resonance wavelength $\lambda_{0, \text { th }}=910.5 \mathrm{~nm}$ on the chiral photonic crystal slab thickness $L$. Blue line in panel (b) shows the calculated circular selectivity of the chiral slab transmission $\rho_{c, T}=\left(T^{+}-T^{-}\right) /\left(T^{+}+T^{-}\right)$; see the discussion in Sec. IV B. the electromagnetic field rotated by $90^{\circ}$ is also a solution. The $90^{\circ}$ rotation switches the linear polarization of the illuminated plane wave $j=x$ to the orthogonal polarization $j=y$, while the integral (4) does not change, $I^{x}=I^{y}$. Because of Eq. (4), $\rho_{x y}=\left(I^{x}-I^{y}\right) /\left(I^{x}+I^{y}\right)=0$, hence the emission cannot be linearly polarized.

Similarly, if a structure has a $D_{4}$ symmetry (equivalent to $C_{4 v}$ ), Maxwell's equations are invariant also under mirror reflection operation, e.g., in the $X O Z$ plane, which switches the sense of circular polarization of the illuminated plane wave $j= \pm$ to the opposite one, $j=\mp$, while the integral (4) does not change, $I^{+}=I^{-}$. Thus, again, the degree of circular polarization of emission $\rho_{c}=\left(I^{+}-I^{-}\right) /\left(I^{+}+I^{-}\right)=0$, hence the emission cannot be circularly polarized, too.

\section{S-matrix approach and $C_{4}$ symmetry}

In the structure of interest with $C_{4}$ symmetry, a layer with randomly distributed and randomly polarized QD is placed in the planar GaAs waveguide. Two lower effective dielectric permittivity layers operate as mirrors. The bottom mirror is the $\mathrm{Al}_{0.7} \mathrm{Ga}_{0.3} \mathrm{As}$ layer. The top mirror is the $2 \mathrm{D}$ chiral photonic crystal. The propagation of light in this system can be described by the optical scattering matrix. The basic idea is (i) to split the system into several layers in which the dielectric permittivity does not depend on $z$, and (ii) to find the $2 \mathrm{D}$ electromagnetic modes of each slab, their electromagnetic field distributions $\left\{E_{x}(x, y), E_{y}(x, y)\right\},\left\{H_{x}(x, y), H_{y}(x, y)\right\}$, and wave numbers $K_{z}$. The periodicity of the structure along $x$ and $y$ axes leads the fields to be represented as products of periodic functions and harmonics $\exp \left[i\left(k_{x} x+k_{y} y\right)\right]$, where $k_{x, y}$ are in the first $\mathrm{BZ}$, with $-K_{\mathrm{BZ}} / 2<k_{x, y}<K_{\mathrm{BZ}} / 2$.

The $C_{4}$ symmetry leads the in-plane components of the electric and magnetic fields to be represented after a $90^{\circ}$ rotation in one of the four forms

$$
\left\{F_{x}(-y, x), F_{y}(-y, x)\right\}=i^{n}\left\{F_{y}(x, y),-F_{x}(x, y)\right\},
$$

where $n=-1,0,1,2$, and $F$ means the electric or magnetic fields. The advantages of the periodicity and $C_{4}$ symmetry can be simultaneously used only for $k_{x}=k_{y}=0$. In this case, the left polarized light $\mathbf{E}^{-}=E \frac{\mathbf{e}_{x}+i \mathbf{e}_{y}}{\sqrt{2}} e^{i k_{0} z-i \omega t}$, propagating in air normal to the structure plane, corresponds to $n=-1$, while the right polarized one corresponds to $n=+1$. Since two other forms are optically inactive (see, e.g., in Ref. [13]), we will consider later only $n= \pm 1$.

\section{Time-reversal symmetry}

The time-reversal symmetry of Maxwell's equations for systems consisting of nonabsorbing and nongyrotropic materials relates wave numbers and electromagnetic fields of the propagating $\left(\operatorname{Im} K_{z}=0\right)$ modes with opposite $n$. If $\mathbf{E}_{3 D}(x, y, z)$ and $\mathbf{H}_{3 D}(x, y, z)$ are the solutions of Maxwell's equations for infinitely thick layer, the time-reversal symmetry requires $\mathbf{E}_{3 D}^{*}(x, y, z)$ and $-\mathbf{H}_{3 D}^{*}(x, y, z)$ to be the solutions, too.

Considering the propagation of some mode with fields $\left\{E_{x}(x, y), E_{y}(x, y)\right\} e^{i K_{z} z} \quad$ and $\quad\left\{H_{x}(x, y), H_{y}(x, y)\right\} e^{i K_{z} z}$, one obtains that $\left\{E_{x}^{*}(x, y), E_{y}^{*}(x, y)\right\} e^{-i K_{z} z}$ and $-\left\{H_{x}^{*}(x, y), H_{y}^{*}(x, y)\right\} e^{-i K_{z} z}$ are also solutions. It corresponds to the counterpropagating mode with opposite polarization $n$, 

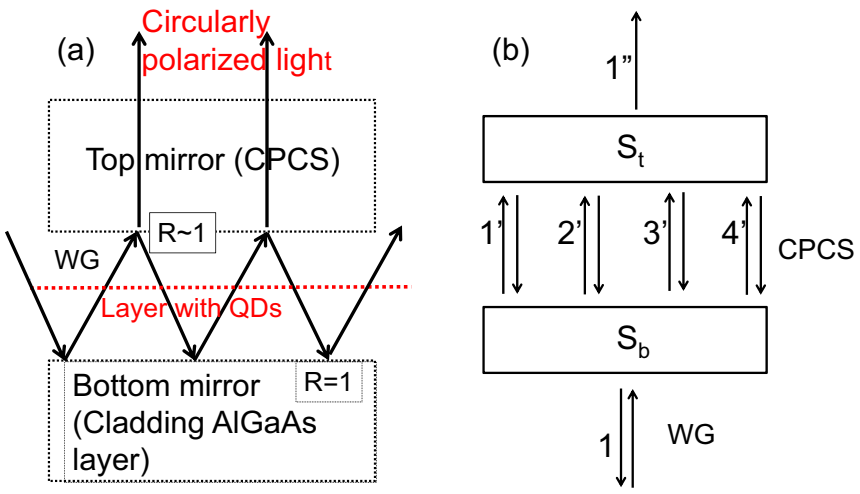

FIG. 10. (Color online) (a) Scheme of light scattering in the structure. (b) Schematic of $\mathbf{k}=0$ light emission near the $\lambda=$ $910.5-\mathrm{nm}$ resonance. Only the propagating modes are shown, with wave numbers (in units of $k_{0}$ ) $K_{z 1^{\prime \prime}}=1 ; K_{z 1^{\prime}, 2^{\prime}, 3^{\prime}, 4^{\prime}}=$ $2.61,1.85,1.32,1.22 ; K_{z 1}=1.11$. Actually, there are 12 more propagating modes in the waveguide layer (including the $k=0, K_{z} / k_{0}=$ $\sqrt{\varepsilon}=3.52$ mode), but in comparison with the quasiguided mode 1 they do not scatter back from the bottom interface of the waveguide even if they are excited on the top interface and thus can be neglected.

since complex conjugation of one polarization $(n=+1$ or $-1)$ switches the polarization to the opposite one $(n=-1$ or +1 , respectively). As a result,

$$
K_{z}^{+}=K_{z}^{-}, E^{+}=\left(E^{-}\right)^{*}, H^{+}=\left(H^{-}\right)^{*},
$$

where the upper indices \pm show the values of $n$. Note that the eigenmodes are always defined up to an arbitrary factor $C$. If propagating eigenmodes are normalized to have fixed energy flow (we choose $\frac{c}{8 \pi}$ per elementary unit cell), the eigenmodes are still defined up to an arbitrary factor with unitary absolute value $e^{i \psi}$. Hereinafter, we choose the phase $\psi$ for the opposite circular polarizations so that their electromagnetic fields are complex conjugates of each other.

\section{Unitary scattering matrix}

The optical scattering matrix can be approximately replaced by the unitary scattering matrix $S_{u}$ [18]. The thicker the chiral photonic crystal layer is, the better is this approximation. The schematic of the QD emission near the $\lambda_{0, \mathrm{th}}=910.5-\mathrm{nm}$ resonance with $n=+1$ or -1 (decoupled in the case of $C_{4}$ symmetry) is shown in Fig. 10(a).

Here, $S_{t}$ and $S_{b}$ are the unitary scattering matrices of the air-CPCS and CPCS-waveguide interfaces, respectively. Hereinafter we omit the subscript $u$ in the scattering matrix notation for simplicity.

In general, the unitary scattering matrix expresses the amplitudes of outgoing modes in terms of the amplitudes of incoming modes:

$$
\left(\begin{array}{c}
\text { out }_{1} \\
\text { out }_{2} \\
\text { out }_{3} \\
\ldots
\end{array}\right)=S\left(\begin{array}{l}
\text { in }_{1} \\
\text { in }_{2} \\
\text { in }_{3} \\
\ldots
\end{array}\right)=\left(\begin{array}{cccc}
r_{1} & t_{12} & t_{13} & . \\
t_{21} & r_{2} & t_{23} & . \\
t_{31} & t_{32} & r_{3} & . \\
\ldots & \ldots & \ldots & .
\end{array}\right)\left(\begin{array}{c}
\mathrm{in}_{1} \\
\mathrm{in}_{2} \\
\text { in }_{3} \\
\ldots
\end{array}\right) .
$$

The diagonal elements of the unitary scattering matrix are the reflection amplitudes $r_{i}$ of the $i$ th channel, and nondiagonal elements are the transition amplitudes $t_{i j}$ from the $j$ th channel to the $i$ th channel.

Similar to the relations in Eq. (6), the time-reversal symmetry of Maxwell's equations for systems consisting of nonabsorbing and nongyrotropic materials relates also reflection and transition amplitudes in $\sigma^{ \pm}$polarizations:

$$
r_{i}^{+}=r_{i}^{-}, t_{i j}^{+}=t_{j i}^{-} \text {. }
$$

\section{Two-mode resonances}

In the structure of interest, at the resonance wavelength, the QDs excite mode 1, which leaks along the $z$ axis primarily through the channels $1^{\prime}$ and $2^{\prime}$ with transition amplitudes $t_{1 \rightarrow 1^{\prime}}^{ \pm}$ and $t_{1 \rightarrow 2^{\prime}}^{ \pm}$, respectively; see Fig. 10(b). For polarizations $\sigma^{+}$ or $\sigma^{-}$, these modes propagate through the CPCS with wave numbers $K_{z 1^{\prime}}$ and $K_{z 2^{\prime}}$, respectively. At the top boundary of the CPCS these modes transform into mode $1^{\prime \prime}$ with the transition amplitudes $t_{1^{\prime} \rightarrow 1^{\prime \prime}}^{ \pm}$and $t_{2^{\prime} \rightarrow 1^{\prime \prime}}^{ \pm}$, respectively. The total transition probability, including the phase shift due to propagation over the CPCS, is

$$
T^{ \pm}=\left|t_{1 \rightarrow 1^{\prime}}^{ \pm} t_{1^{\prime} \rightarrow 1^{\prime \prime}}^{ \pm} e^{i K_{z 1^{\prime}} L}+t_{1 \rightarrow 2^{\prime}}^{ \pm} t_{2^{\prime} \rightarrow 1^{\prime \prime}}^{ \pm} e^{i K_{z 2^{\prime}} L}\right|^{2},
$$

where $L$ is the thickness of the CPCS. To simplify the formulas, let us introduce the new variables

$$
\begin{gathered}
T_{0}^{ \pm}=4\left|t_{1 \rightarrow 1^{\prime}}^{ \pm} t_{1^{\prime} \rightarrow 1^{\prime \prime}}^{ \pm}\right|^{2}, \\
\eta^{ \pm}=\left|\frac{t_{1 \rightarrow 2^{\prime}}^{ \pm} t_{2^{\prime} \rightarrow 1^{\prime \prime}}^{ \pm}}{t_{1 \rightarrow 1^{\prime}}^{ \pm} t_{1^{\prime} \rightarrow 1^{\prime \prime}}^{ \pm}}\right|, \\
\varphi^{ \pm}=\arg \left(\frac{t_{1 \rightarrow 2^{\prime}}^{ \pm} t_{2^{\prime} \rightarrow 1^{\prime \prime}}^{ \pm}}{t_{1 \rightarrow 1^{\prime}}^{ \pm} t_{1^{\prime} \rightarrow 1^{\prime \prime}}^{ \pm}}\right),
\end{gathered}
$$
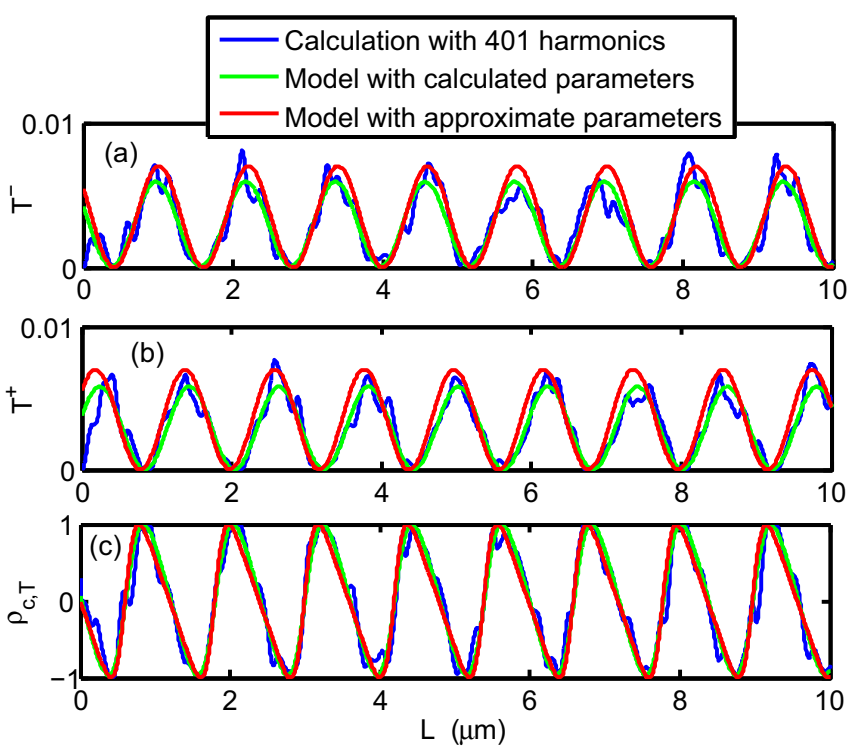

FIG. 11. (Color online) The calculated dependencies of the CPCS transmission $T^{ \pm}$in (a) right and (b) left polarizations on CPCS thickness $L$. Panel (c) shows the calculated circular selectivity of transmission $\rho_{c, T}$. Blue lines are the results of calculations with 401 spatial harmonics. Green and red lines correspond to the two-modes model Eq. (14) with approximated and calculated (using 401 harmonics) parameters (see in Table I), respectively. 
TABLE I. Comparison of the simple model in the Appendix with the numerical simulation with 401 harmonics.

\begin{tabular}{lccc}
\hline \hline Variable & Approximate & Simulation & Relative difference $^{\mathrm{a}}$ \\
\hline$t_{1 \rightarrow 1^{\prime}}^{+}$ & $-0.0256+0.0830 i$ & $0.0093+0.0673 i$ & $25 \%$ \\
$t_{1 \rightarrow 1^{\prime}}^{-}$ & $-0.0256-0.0830 i$ & $-0.0487-0.0564 i$ & $22 \%$ \\
$t_{1 \rightarrow 2^{\prime}}^{+}$ & $-0.0830+0.0256 i$ & $-0.0452+0.0220 i$ & $28 \%$ \\
$t_{1 \rightarrow 2^{\prime}}^{-}$ & $-0.0830-0.0256 i$ & $-0.0417+0.0199 i$ & $46 \%$ \\
$t_{1^{\prime}}^{+}$ & 0.4820 & $0.5774-0.0613 i$ & $11 \%$ \\
$t_{1^{\prime}}^{-}$ & 0.4820 & $0.5734-0.0994 i$ & $13 \%$ \\
$t_{2^{\prime}}^{+}$ & 0.4820 & $0.7290-0.0561 i$ & $21 \%$ \\
$t_{2^{\prime} \rightarrow 1^{\prime \prime}}^{-}$ & 0.4820 & $0.7277-0.0122 i$ & $20 \%$ \\
$T_{0}^{+}$ & 0.007 & 0.0062 & $6 \%$ \\
$T_{0}^{-}$ & 0.007 & 0.0075 & $4 \%$ \\
$\eta^{+}$ & 1 & 0.932 & $4 \%$ \\
$\eta^{-}$ & 1 & 0.775 & $13 \%$ \\
$\left|\varphi^{+}-\varphi^{-}\right|$ & $111^{\circ}$ & $139^{\circ}$ & $11 \%$ \\
\hline \hline
\end{tabular}

${ }^{\text {a Here, we calculate the relative difference } \delta \text { of two variables } a \text { and } b \text { as } \delta=\frac{|a-b|}{|a|+|b|}}$.

$\Delta K=K_{z 2^{\prime}}-K_{z 1^{\prime}}$. Then

$$
T^{ \pm}=\frac{T_{0}^{ \pm}}{4}\left|1+\eta^{ \pm} e^{i \Delta K L+\varphi^{ \pm}}\right|^{2} .
$$

If $\eta^{ \pm}=1$, then

$$
T^{ \pm}=T_{0}^{ \pm} \cos ^{2} \frac{\Delta K L+\varphi^{ \pm}}{2}
$$

is the oscillating function of the thickness $L$, which varies from 0 to $T_{0}^{ \pm}$. Moreover, if $\mid \varphi^{+}-\varphi^{-}=\pi$, the transmission in one polarization reaches its maximum when the transmission in the opposite polarization reaches the minimum and vice versa. The circular polarization selectivity of transmission $\rho_{c, T}=\left(T^{+}-\right.$ $\left.T^{-}\right) /\left(T^{+}+T^{-}\right)$in these thicknesses appears to be around $100 \%$.

A simple model to estimate the introduced above parameters for the resonance at $E_{0}$ is given in the Appendix. The results of the simplified model are compared with the optical scattering matrix calculation in Fig. 11. Although the parameters of the simplified model seem to differ substantially from the ones calculated within the scattering matrix method with 401 harmonics (see in Table I), the results from this crude model for the circular polarization selectivity of transmission [Fig. 11(c)] are in a good quantitative agreement with the calculations within the scattering matrix. It happens because $\eta^{ \pm},\left|\varphi^{+}-\varphi^{-}\right|$and especially the $\rho_{c}$ are sensitive to the ratios of the partial transmissions trough modes $1^{\prime}$ and $2^{\prime}$, which deviate less from the calculated values.

The dependence $\rho_{c, T}(L)$ is also shown as blue line in Fig. 9(b). It can be seen that it is very close to the dependence $\rho_{c}(L)$ (green line in the same panel). This fact supports our assumption that it is the selectivity of transmission through the chiral photonic slab that controls the degree of circular polarization of QD emission in our structure.

\section{CONCLUSION}

In conclusion, we have fabricated and studied a structure with light emitting InAs QDs inside a GaAs waveguide with a chiral photonic crystal layer on top that demonstrates a strongly circularly polarized photoemission of QDs at zero magnetic field with $\left|\rho_{c}\right|$ up to $81 \%$ and up to $96 \%$ if the unpolarized background due to CPCS imperfection is removed. It indicates that the predicted values of $\left|\rho_{c}\right|$ close to $100 \%$ can be reached with further progress in fabrication technology.

The measured angular dependence of the circularly polarized emission intensities near the resonance is shown to agree with the calculations using the optical scattering matrix. This allows us to attribute the circularly polarized resonant emission to the excitation by QDs of the $( \pm 2, \pm 2)$ Bragg resonance of quasiguided modes in the GaAs waveguide. Based on the reciprocity and symmetry analysis of the structure, we show that the chiral photonic crystal slab in the suggested waveguide structure works as a half-wave plate, exploring the Fabry-Perot interference between the propagating modes in CPCS, to reach nearly a $100 \%$ circular polarization of the transmission. The advantage of using the photonic crystal slab with a large contrast of dielectric permittivities is its giant chirality, which allows us to fabricate a very thin half-wave plate, with a thickness of the order of the emitted light wavelength. An additional advantage of the CPCS wave plate is its in-plane rotational isotropy, due to the $C_{4}$ symmetry.

\section{ACKNOWLEDGMENTS}

This work has been funded by the project SPANGL4Q, under FET-Open Grant No. FP7-284743, and RFBR Projects No. 13-02-12144 and No. 14-02-00778. We are grateful to K. Konishi, L. Kuipers, M. Kuwata-Gonokami, R. Oulton, and H. Tamaru for fruitful discussions. We thank M. Emmerling for preparing the nanopillars, and acknowledge support by the state of Bavaria as well as the MWK Baden, Württemberg.

\section{APPENDIX: ANALYTICAL ESTIMATION OF TRANSITION AMPLITUDES}

Here we investigate the resonance shown in Fig. 4 in the optimized structure and assume the free space wavelength 
of light to be fixed and equal to $910.5 \mathrm{~nm}$. The transition amplitudes $t_{i \rightarrow j}$ in Eqs. (10)-(12) can be roughly estimated via the normalized [see after Eq. (6)] in-plane components of the electric and magnetic fields of modes $i, j$ as

$$
t_{i \rightarrow j}=\iint_{-p / 2}^{p / 2} d x d y \frac{\mathbf{E}_{j}^{*} \times \mathbf{H}_{i}+\mathbf{E}_{i} \times \mathbf{H}_{j}^{*}}{2} .
$$

Here, the symbol $\times$ means the pseudoscalar product

$$
\mathbf{E} \times \mathbf{H}=E_{x} H_{y}-E_{y} H_{x} .
$$

The CPCS consists of dielectric rectangular pillars that are known to support the waveguide modes (in the vertical direction). The electric(magnetic)-field distribution of the waveguide mode with the larger/smaller value of wave number $K_{z}$ can be roughly approximated by a homogeneous field parallel to the longest/shortest (shortest/longest) side inside the pillar and zero field outside it. Considering four rectangular pillars each rotated by $90^{\circ}$ with respect to its nearest neighbors requires the electric and magnetic fields to satisfy Eq. (5). Note that, due to the condition (5), the integral in Eq. (A1) can be taken only over the first quarter of the elementary unit cell (it should be also multiplied by 4$)$, since $\mathbf{E}^{*}(-y, x) \times \mathbf{H}(-y, x)=$ $\mathbf{E}^{*}(x, y) \times \mathbf{H}(x, y)$.

The electric- and magnetic-field distributions of modes $1^{\prime}$ and $2^{\prime}$ in the first quarter of the elementary unit cell are

$$
\begin{aligned}
& \mathbf{E}_{1^{\prime}}^{ \pm}(x, y)= \begin{cases}\mp i C_{1} \varepsilon^{-1 / 4} \mathbf{e}_{y}, & \text { if } x \in\left(\frac{p}{5}, \frac{2 p}{5}\right) \& y \in\left(0, \frac{2 p}{5}\right) \\
0, & \text { otherwise, }\end{cases} \\
& \mathbf{H}_{1^{\prime}}^{ \pm}(x, y)= \begin{cases} \pm i C_{1} \varepsilon^{1 / 4} \mathbf{e}_{x}, & \text { if } x \in\left(\frac{p}{5}, \frac{2 p}{5}\right) \& y \in\left(0, \frac{2 p}{5}\right) \\
0, & \text { otherwise, }\end{cases} \\
& \mathbf{E}_{2^{\prime}}^{ \pm}(x, y)= \begin{cases}C_{1} \varepsilon^{-1 / 4} \mathbf{e}_{x}, & \text { if } x \in\left(\frac{p}{5}, \frac{2 p}{5}\right) \& y \in\left(0, \frac{2 p}{5}\right) \\
0, & \text { otherwise, }\end{cases} \\
& \mathbf{H}_{2^{\prime}}^{ \pm}(x, y)= \begin{cases}C_{1} \varepsilon^{1 / 4} \mathbf{e}_{y}, & \text { if } x \in\left(\frac{p}{5}, \frac{2 p}{5}\right) \& y \in\left(0, \frac{2 p}{5}\right) \\
0, & \text { otherwise, }\end{cases}
\end{aligned}
$$

where the constant $C_{1}$ is introduced for brevity,

$$
C_{1}=\frac{5}{2 \sqrt{2} p} .
$$

The electric- and magnetic-field distributions of modes 1 [which is the $( \pm 2, \pm 2)$ Bragg resonance of the GaAs layer guided mode, in the horizontal direction] and $1^{\prime \prime}$ are

$$
\begin{aligned}
\mathbf{E}_{1}^{ \pm}(x, y)= & \frac{1}{p} \sqrt{\frac{k_{0}}{K_{z 1}}}\left[\frac{-\mathbf{e}_{x}+\mathbf{e}_{y}}{\sqrt{2}} \cos \left(\frac{4 \pi}{p}(x+y)\right)\right. \\
& \left. \pm i \frac{\mathbf{e}_{x}+\mathbf{e}_{y}}{\sqrt{2}} \cos \left(\frac{4 \pi}{p}(x-y)\right)\right] \\
\mathbf{H}_{1}^{ \pm}(x, y)= & \frac{1}{p} \sqrt{\frac{K_{z 1}}{k_{0}}}\left[-\frac{\mathbf{e}_{x}+\mathbf{e}_{y}}{\sqrt{2}} \cos \left(\frac{4 \pi}{p}(x+y)\right)\right. \\
& \left. \pm i \frac{-\mathbf{e}_{x}+\mathbf{e}_{y}}{\sqrt{2}} \cos \left(\frac{4 \pi}{p}(x-y)\right)\right]
\end{aligned}
$$

$$
\begin{aligned}
& \mathbf{E}_{1^{\prime \prime}}^{ \pm}(x, y)=\frac{1}{p} \frac{\mathbf{e}_{x} \mp i \mathbf{e}_{y}}{\sqrt{2}}, \\
& \mathbf{H}_{1^{\prime \prime}}^{ \pm}(x, y)=\frac{1}{p} \frac{\mathbf{e}_{y} \pm i \mathbf{e}_{x}}{\sqrt{2}},
\end{aligned}
$$

where the wave number is

$$
K_{z 1}=\sqrt{\varepsilon k_{0}^{2}-\frac{32 \pi^{2}}{p^{2}}} ;
$$

$\varepsilon=12.42$ is the GaAs dielectric permittivity.

Substituting (A3)-(A10) in (A1), one can find the transition amplitudes

$$
\begin{gathered}
t_{1 \rightarrow 1^{\prime}}^{ \pm}=C_{2}\left(-C_{3} \pm i C_{4}\right), \\
t_{1 \rightarrow 2^{\prime}}^{ \pm}=C_{2}\left(-C_{4} \pm i C_{3}\right), \\
t_{1^{\prime} \rightarrow 1^{\prime \prime}}^{ \pm}=t_{2^{\prime} \rightarrow 1^{\prime \prime}}^{ \pm}=C_{5},
\end{gathered}
$$

where the constants $C_{2}, C_{3}, C_{4}$, and $C_{5}$ are

$$
\begin{gathered}
C_{2}=\frac{5}{16 \pi^{2}}\left(\varepsilon^{1 / 4} \sqrt{\frac{k_{0}}{K_{z 1}}}+\varepsilon^{-1 / 4} \sqrt{\frac{K_{z 1}}{k_{0}}}\right), \\
C_{3}=\sin ^{2} \frac{\pi}{5}, \\
C_{4}=\cos \frac{2 \pi}{5}+\cos \frac{\pi}{5}, \\
C_{5}=\frac{1}{5}\left(\varepsilon^{1 / 4}+\varepsilon^{-1 / 4}\right) .
\end{gathered}
$$

Substituting formulas (A12)-(A14) into the definitions of $T_{0}^{ \pm}, \eta^{ \pm}$, and $\varphi^{ \pm}$, i.e., Eqs. (10), (11), and (12), respectively, one finds

$$
\begin{gathered}
T_{0}^{ \pm}=C_{2}^{2} C_{5}^{2}\left(C_{3}^{2}+C_{4}^{2}\right) \approx 0.007, \\
\eta^{ \pm}=1, \\
\left|\varphi^{+}-\varphi^{-}\right|=\pi-4 \operatorname{atan} \frac{C_{3}}{C_{4}} \approx 111^{\circ} .
\end{gathered}
$$

Thus, as discussed above in Eq. (14), transmission in each polarization depends periodically on the CPCS thickness. As the function of thickness, it varies from 0 to 0.007 and is shifted by $111^{\circ}$ with respect to the transmission in the opposite polarization. The comparison of this simple model with the numerical simulation with 401 harmonics is given in Table I. The resulting dependencies of the CPCS transmission in $\sigma^{+}$ and $\sigma^{-}$polarizations and of the circular polarization degree of transmission in comparison with the same formulas (but using exact parameters from Table I) and the results of simulation with 401 harmonics are given in Fig. 11. It can be seen that this very approximate and crude model is in qualitative agreement with the numerical simulations. The dependencies in Fig. 11, calculated with 401 harmonics, demonstrate also sharp peaks, which are due to the influence of other electromagnetic modes in the CPCS, not taken into account in this simple two-mode model [19]. 
[1] H. Stegemeyer, W. Stille, and P. Pollmann, Israel J. Chem. 18, 312 (1979).

[2] J. Schmidtke and W. Stille, Eur. Phys. J. B 31, 179 (2003).

[3] K. L. Woon, M. O’Neill, G. J. Richards, M. P. Aldred, and S. M. Kelly, Phys. Rev. E 71, 041706 (2005).

[4] I. De Leon, M. J. Horton, S. A. Schulz, J. Upham, P. Banzer, and R. W. Boyd, Sci. Rep. 5, 13034 (2015).

[5] K. Konishi, M. Nomura, N. Kumagai, S. Iwamoto, Y. Arakawa, and M. Kuwata-Gonokami, Phys. Rev. Lett. 106, 057402 (2011).

[6] N. Shitrit, I. Yulevich, E. Maguid, D. Ozeri, D. Veksler, V. Kleiner, and E. Hasman, Science 340, 724 (2013).

[7] L. D. Barron, Nature (London) 238, 17 (1972).

[8] A. A. Maksimov, I. I. Tartakovskii, E. V. Filatov, S. V. Lobanov, N. A. Gippius, S. G. Tikhodeev, C. Schneider, M. Kamp, S. Maier, S. Höfling, and V. D. Kulakovskii, Phys. Rev. B 89, 045316 (2014).

[9] S. V. Lobanov, T. Weiss, N. A. Gippius, S. G. Tikhodeev, V. D. Kulakovskii, K. Konishi, and M. Kuwata-Gonokami, Opt. Lett. 40, 1528 (2015).

[10] S. V. Lobanov, T. Weiss, D. Dregely, H. Giessen, N. A. Gippius, and S. G. Tikhodeev, Phys. Rev. B 85, 155137 (2012).
[11] I. Söllner, S. Mahmoodian, S. L. Hansen, L. Midolo, A. Javadi, G. Kiršanskè, T. Pregnolato, H. El-Ella, E. H. Lee, J. D. Song, S. Stobbe, and P. Lodahl, Nat. Nanotechnol. 10, 775 (2015).

[12] The sign of circular polarization is defined from the point of view of the receiver. The electric field of a planar left $\left(\sigma^{-}\right)$ circularly polarized wave, propagating in the $z$ direction and rotating counterclockwise from the point of view of the receiver is $E^{-} \propto\left(\mathbf{e}_{x}+i \mathbf{e}_{y}\right) \exp \left(i k_{0} z-i \omega t\right)$.

[13] S. G. Tikhodeev, A. L. Yablonskii, E. A. Muljarov, N. A. Gippius, and T. Ishihara, Phys. Rev. B 66, 045102 (2002).

[14] D. M. Whittaker and I. S. Culshaw, Phys. Rev. B 60, 2610 (1999).

[15] D. M. Whittaker, Opt. Lett. 25, 779 (2000).

[16] H. Taniyama and M. Notomi, J. Appl. Phys. 103, 083115 (2008).

[17] L. D. Landau, L. P. Pitaevskii, and E. M. Lifshitz, Electrodynamics of Continuous Media, 2nd ed. (Elsevier, New York, 1984), Vol. 8, Chap. 89.

[18] N. A. Gippius, S. G. Tikhodeev, and T. Ishihara, Phys. Rev. B 72, 045138 (2005).

[19] See Supplemental Material at http://link.aps.org/supplemental/ 10.1103/PhysRevB.92.205309 for more details on the comparison of the model with numerical simulations. 